\title{
Higher Order Multi-Dimensional Extensions of Cesàro Theorem
}

\author{
Luigi ACCARDi \\ Centro Vito Volterra Facultà di Economica \\ UNIVERSITÀ DI TOR VERGATA \\ Via di Tor Vergata, 00133 Roma, Italy \\ E-MAIL: accardi@volterra.mat.uniroma.it \\ Un Cig Ji \\ Department of MATHEMATiCs \\ Research Institute of Mathematical Finance \\ Chungbuk National University \\ Cheongue 361-763, Korea \\ E-MAIL: uncigji@chungbuk.ac.kr \\ AND \\ KIMIAKI SAITÔ \\ Department of MATHEMATiCS \\ MEIJO UNIVERSITY \\ NAGOYA 468, JAPAN \\ E-MAIL: ksaito@meijo-u.ac.jp
}

\begin{abstract}
The Cesàro theorem is extended to the cases: (1) higher order Cesàro mean for sequence (discrete case); and (2) higher order, multi-dimensional and continuous Cesàro mean for functions. Also, we study the Cesàro theorem for the case of positive-order.
\end{abstract}

Keywords: Cesàro mean, positive-order Cesàro mean, Cesàro theorem, continuous Cesàro theorem, higher order Cesàro theorem

Mathematics Subject Classification (2000): 40G05. 


\section{Introduction}

It is known that the Lévy Laplacian [17] is an infinite dimensional Laplacian that can be defined as a Cesàro mean of order 1 of the second derivatives along the elements of an orthonormal basis of a Hilbert space. Motivated by the paper [2] this construction was generalized in [8] to higher order extension of Cesàro means, leading to the notion of exotic Laplacian. The solution of the heat equation for the hierarchy of exotic Laplacians was first obtained in the paper [3].

In [4] it was proved that all exotic Laplacians can be realized in appropriate completions of subspaces of the Hida distribution space, thus showing that this space plays a fundamental role not only for the Lévy Laplacian, but for the whole exotic hierarchy. Finally, in [5] the Markov process generated by the exotic Laplacian of order $2 a$ was identified to the Brownian motion associated of the $a$-th distribution derivative of the standard white noise, thus providing a natural probabilistic interpretation for the exotic Laplacians. In fact, after this result, the term exotic seems no longer justified, since these are natural expressions of a fundamental mathematical object such as the standard white noise.

The above mentioned identifications were made possible, on one side by a generalization to higher order means, of the known Cesàro's theorems on the arithmetic mean (see Section 5), on the other side, by the formulation and proof of the inverses of these results. This generalization was achieved in successive steps in increasing order of generality: the first result, obtained in [10], concerned sequences (as in the original Cesàro theorem) and means of integer order. The second result in [10] concerns the converse of the first one: this seems to be a new type of Cesàro theorems, not previously considered in the literature. Both results played a crucial role in the construction, given in [4] of a similarity relation among exotic Laplacians of order $\geq 1$. This was extended in [5] to sequences and means of arbitrary real order. Finally, in the present paper, sequences are replaced by arbitrary functions on $\mathbb{R}^{d}$. Such an extension is required in order to bring white noise theory nearer to the quantum field theory formalism and constitutes a first non-trivial step in this direction.

This paper is organized as follows: In Section 2 we establish a continuous multidimensional extension of Cesàro theorem for positive higher order. In Section 3 we prove one of our main result concerning the higher order, multi-dimensional and continuous extension of Cesàro theorem. In Section 4 we prove a converse version of the higher order Cesàro theorem studied in Section 3. In Section 5 we introduce a construction that allows to reduce all Cesàro type theorems to the corresponding results in the discrete 1-dimensional case for sequences.

Acknowledgements. LA acknowledges support by the RSF grant 14-11-00687. UCJ was supported by the research grant of the Chungbuk National University in 2012. KS was supported by JSPS-NRF under the Japan-Korea Basic Scientific Cooperation Program 2015-2016 and the JSPS grant 15K04940. 


\section{Positive Order Cesàro Theorems: Continuous Case}

In this section, we study positive order Cesàro theorems for functions on multidimensional Euclidean space.

The Euclidean distance in $\mathbb{R}^{d}$ is denoted by $\operatorname{dist}(\cdot, \cdot)$ and, for any $r>0$,

$$
B(x, r):=\left\{y \in \mathbb{R}^{d}: \operatorname{dist}(x, y)<r\right\}
$$

is the open ball in $\mathbb{R}^{d}$, centered in $x \in \mathbb{R}^{d}$, with radius $r$ and $B(x, r)^{\mathrm{c}}$ its complement. In this section we define the generalized Cesàro means as linear functionals defined on some vector subspaces of $L_{\text {loc }}^{1}\left(\mathbb{R}^{d}\right)$, the space of all locally integrable functions on $\mathbb{R}^{d}$, and we prove some properties of these means. We start with the Cesàro mean of order 1 , correpsonding to the original version of Cesàro theorem. For a given $p \in \mathbb{R}_{+}$, the linear functional $C_{p}$ defined by:

$$
\begin{gathered}
\operatorname{Dom}\left(C_{p}\right):=\left\{g \in L_{\mathrm{loc}}^{1}\left(\mathbb{R}^{d}\right): \lim _{r \rightarrow \infty} \frac{1}{|B(0, r)|^{p}} \int_{B(0, r)} g(k) d k \text { exists }\right\} \\
C_{p}(g):=\lim _{r \rightarrow \infty} \frac{1}{|B(0, r)|^{p}} \int_{B(0, r)} g(k) d k
\end{gathered}
$$

is called the Cesàro mean of order $p$ of $g \in \operatorname{Dom}\left(C_{p}\right)$. The Cesàro mean of order 1 is simply called the Cesàro mean.

Remark 2.1. In the case $d=1$,

$$
B(0, r)=(-r, r)
$$

hence (2.2) becomes, for $p=1$ :

$$
C_{1}(g):=\lim _{r \rightarrow \infty} \frac{1}{2 r} \int_{-r}^{r} g(t) d t
$$

for $g \in \operatorname{Dom}\left(C_{1}\right)$, which is slightly different from the usual definition of Cesàro mean

$$
C_{1}(g):=\lim _{r \rightarrow \infty} \frac{1}{r} \int_{0}^{r} g(t) d t
$$

for $g \in \operatorname{Dom}\left(C_{1}\right)$ that only considers the interval $(0, r)$. For the continuous and multidimensional extensions, the symmetric formulation has some advantages.

Theorem 2.2. Let $g \in L_{\mathrm{loc}}^{1}\left(\mathbb{R}^{d}\right)$ be such that the limit

$$
\lim _{|t| \rightarrow \infty} \frac{g(t)}{\left(1+|t|^{2}\right)^{p d}}=\lim _{|t| \rightarrow \infty} \frac{g(t)}{|t|^{2 p d}}=C
$$

exists for some $p \geq 0$. Then:

$$
C_{2 p+1}(g)=\frac{1}{|B(0,1)|^{2 p}} \frac{C}{(2 p+1)}
$$

in the sense that the left hand side exists and is equal to the right hand side. 
Proof. It is clear that the two limits in (2.5) are equal in the sense that, one exists if and only if the other one does and in this case equality holds. Assumption (2.5) implies that, for any $\epsilon>0$, there exists $r_{\epsilon} \in \mathbb{R}_{+}$such that, if $|t| \geq r_{\epsilon}$, then

$$
C-\epsilon \leq \frac{g(t)}{|t|^{2 p d}} \leq C+\epsilon \quad \text { or equivalently } \quad(C-\epsilon)|t|^{2 p d} \leq g(t) \leq(C+\epsilon)|t|^{2 p d}
$$

Therefore, for all $r>r_{\epsilon}$ we obtain that

$$
\begin{aligned}
(C-\epsilon) \frac{1}{|B(0, r)|^{2 p+1}} \int_{B(0, r) \backslash B\left(0, r_{\epsilon}\right)}|t|^{2 p d} d t & \leq \frac{1}{|B(0, r)|^{2 p+1}} \int_{B(0, r) \backslash B\left(0, r_{\epsilon}\right)} g(t) d t \\
& \leq(C+\epsilon) \frac{1}{|B(0, r)|^{2 p+1}} \int_{B(0, r) \backslash B\left(0, r_{\epsilon}\right)}|t|^{2 p d} d t
\end{aligned}
$$

Clearly

$$
\frac{1}{|B(0, r)|^{2 p+1}} \int_{B\left(0, r_{\epsilon}\right)}|t|^{2 p d} d t \leq \frac{1}{|B(0, r)|^{2 p+1}} r_{\epsilon}^{2 p d} \int_{B\left(0, r_{\epsilon}\right)} d t=\frac{r_{\epsilon}^{2 p d}\left|B\left(0, r_{\epsilon}\right)\right|}{|B(0, r)|^{2 p+1}}
$$

which tends to 0 as $r \rightarrow \infty$. Put

$$
c_{\epsilon}(r):=\frac{1}{|B(0, r)|^{2 p+1}} \int_{B\left(0, r_{\epsilon}\right)}|t|^{2 p d} d t
$$

Then from the known formula:

$$
|B(0, r)|=\frac{\pi^{d / 2} r^{d}}{\Gamma\left(\frac{d}{2}+1\right)}=|B(0,1)| r^{d}
$$

we obtain that

$$
\begin{aligned}
\frac{1}{|B(0, r)|^{2 p+1}} \int_{B(0, r) \backslash B\left(0, r_{\epsilon}\right)}|t|^{2 p d} d t & =\frac{1}{|B(0,1)|^{2 p+1}} \frac{1}{r^{(2 p+1) d}} \int_{B(0, r) \backslash B\left(0, r_{\epsilon}\right)}|t|^{2 p d} d t \\
& =\frac{1}{|B(0,1)|^{2 p+1}} \frac{1}{r^{(2 p+1) d}} \int_{B(0, r)}|t|^{2 p d} d t-c_{\epsilon}(r) \\
& =\frac{d}{|B(0,1)|^{2 p}} \frac{1}{r^{(2 p+1) d}} \int_{0}^{r} s^{d-1} s^{2 p d} d s-c_{\epsilon}(r)
\end{aligned}
$$

which implies that

$$
\frac{1}{|B(0, r)|^{2 p+1}} \int_{B(0, r) \backslash B\left(0, r_{\epsilon}\right)}|t|^{2 p d} d t=\frac{1}{|B(0,1)|^{2 p}} \frac{1}{(2 p+1)}-c_{\epsilon}(r)
$$

From this it follows that, since $\lim _{r \rightarrow \infty} c_{\epsilon}(r)=0$, by taking limit as $r \rightarrow \infty$ in (2.7),

$$
\begin{aligned}
(C-\epsilon) \frac{1}{|B(0,1)|^{2 p}} \frac{1}{(2 p+1)} & \leq \liminf _{r \rightarrow \infty} \frac{1}{|B(0, r)|^{2 p+1}} \int_{B(0, r) \backslash B\left(0, r_{\epsilon}\right)} g(t) d t \\
\leq \limsup _{r \rightarrow \infty} \frac{1}{|B(0, r)|^{2 p+1}} \int_{B(0, r) \backslash B\left(0, r_{\epsilon}\right)} g(t) d t & \leq(C+\epsilon) \frac{1}{|B(0,1)|^{2 p}} \frac{1}{(2 p+1)}
\end{aligned}
$$


Since $\epsilon>0$ is arbitrary and $\lim _{r \rightarrow \infty} \frac{1}{|B(0, r)|^{2 p+1}} \int_{B\left(0, r_{\varepsilon}\right)} g(t) d t=0$, it follows that

$$
\lim _{r \rightarrow \infty} \frac{1}{|B(0, r)|^{2 p+1}} \int_{B(0, r)} g(t) d t=\frac{1}{|B(0,1)|^{2 p}} \frac{C}{(2 p+1)}
$$

in the sense that the limit on the left hand side exists and the identity holds. This proves $(2.6)$.

By taking $p=0$ in Theorem 2.2, we have the following multi-dimensional continuous Cesàro theorem.

Theorem 2.3. If $g \in L_{\text {loc }}^{1}\left(\mathbb{R}^{d}\right)$ is such that the limit

$$
\lim _{|t| \rightarrow \infty} g(t)=: g_{\infty}
$$

exists in $\mathbb{C}$, then

$$
C_{1}(g)=\lim _{r \rightarrow+\infty} \frac{1}{|B(0, r)|} \int_{B(0, r)} g(s) d s=: g_{\infty}
$$

in the sense that the limit exists and the equality holds.

\section{Higher Order Cesàro Theorems: Continuous Case}

In this section, we study higher order Cesàro theorems for functions on multidimensional Euclidean space.

Theorem 3.1. If, for some $p \in \mathbb{R}_{+}$and some $g \in L_{\text {loc }}^{1}\left(\mathbb{R}^{d}\right)$, the limit

$$
\lim _{r \rightarrow \infty} \frac{1}{|B(0, r)|^{p}} \int_{B(0, r)} g(t) d t=C_{p}(g)
$$

exists in $\mathbb{C}$, then for any $a \in \mathbb{R}_{+}^{*}=\mathbb{R}_{+} \backslash\{0\}$, it holds that

$$
\lim _{r \rightarrow \infty} \frac{1}{|B(0, r)|^{p+a}} \int_{B(0, r)}|t|^{a d} g(t) d t=\frac{1}{|B(0,1)|^{a}} \frac{p}{p+a} C_{p}(g)
$$

in the sense that the limit exists and the equality holds.

Proof. Let $g \in \operatorname{Dom}\left(C_{p}\right)$ and $p \in \mathbb{R}_{+}$. If $p>0$, for some $R>0$ we consider the function

$$
g_{R}(t)=\left\{\begin{array}{cl}
g(t) & \text { if }|t| \geq R \\
0 & \text { if }|t|<R
\end{array}\right.
$$

If $p=0$ we regard $g_{R}(t)$ as $g(t)$. Then we can check that $g_{R} \in L_{\text {loc }}^{1}\left(\mathbb{R}^{d}\right), C_{p}(g)=C_{p}\left(g_{R}\right)$ and

$$
\lim _{r \rightarrow \infty} \frac{1}{|B(0, r)|^{p+a}} \int_{B(0, r)}|t|^{a d} g(t) d t=\lim _{r \rightarrow \infty} \frac{1}{|B(0, r)|^{p+a}} \int_{B(0, r)}|t|^{a d} g_{R}(t) d t
$$


in the sense that, if there exists one side of the equality, then there exists another side. Therefore we may prove (3.2) for $g_{R}$. We also have

$$
\begin{aligned}
\frac{1}{|B(0, r)|^{p+a}} \int_{B(0, r)}|t|^{a d} g_{R}(t) d t= & \frac{1}{|B(0, r)|^{p+a}} r^{a d} \int_{B(0, r)} g_{R}(t) d t \\
& -\frac{1}{|B(0, r)|^{p+a}} \int_{B(0, r)}\left(r^{a d}-|t|^{a d}\right) g_{R}(t) d t
\end{aligned}
$$

On the one hand, by using the identity $|B(0, r)|=|B(0,1)| r^{d}$, we obtain that

$$
\begin{aligned}
\frac{1}{|B(0, r)|^{p+a}} r^{a d} \int_{B(0, r)} g_{R}(t) d t & =\frac{1}{|B(0,1)|^{p+a} r^{p d}} \int_{B(0, r)} g_{R}(t) d t \\
& =\frac{1}{|B(0,1)|^{a}} \frac{1}{|B(0, r)|^{p}} \int_{B(0, r)} g_{R}(t) d t
\end{aligned}
$$

and assumption (3.2) implies that the limit of the right hand side for $r \rightarrow \infty$ exists and is equal to

$$
\frac{1}{|B(0,1)|^{a}} C_{p}\left(g_{R}\right)
$$

On the other hand, in the second term of the difference in (3.3), by using $d$-dimensional spherical coordinate representation, we have

$$
\int_{B(0, r)}\left(r^{a d}-|t|^{a d}\right) g_{R}(t) d t=\int_{0}^{r}\left(r^{a d}-s^{a d}\right) s^{d-1}\left(\int_{\Theta} \hat{g_{R}}(s, \hat{\theta}) d \hat{\theta}\right) d s
$$

for some function $\hat{g_{R}}$ induced by $g_{R}$ via $d$-dimensional spherical coordinate representation, where $\Theta=[0, \pi]^{d-2} \times[0,2 \pi]$. Then, by using the identity $|B(0, r)|=|B(0,1)| r^{d}$ again, we obtain that

$$
\begin{aligned}
& \frac{1}{|B(0, r)|^{p+a}} \int_{B(0, r)}\left(r^{a d}-|t|^{a d}\right) g_{R}(t) d t \\
& \quad=\frac{1}{|B(0,1)|^{p+a} r^{(p+a) d}} \int_{0}^{r}\left(r^{a d}-s^{a d}\right) s^{d-1}\left(\int_{\Theta} \hat{g_{R}}(s, \hat{\theta}) d \hat{\theta}\right) d s \\
& \quad=\frac{a d}{|B(0,1)|^{p+a} r^{(p+a) d}} \int_{0}^{r} s^{d-1}\left(\int_{s}^{r} \tau^{a d-1} d \tau\right)\left(\int_{\Theta} \hat{g_{R}}(s, \hat{\theta}) d \hat{\theta}\right) d s \\
& \quad=\frac{a d}{|B(0,1)|^{p+a} r^{(p+a) d}} \int_{0}^{r}\left[\int_{0}^{\tau} s^{d-1}\left(\int_{\Theta} \hat{g_{R}}(s, \hat{\theta}) d \hat{\theta}\right) d s\right] \tau^{a d-1} d \tau
\end{aligned}
$$

which becomes

$$
\begin{aligned}
= & \frac{a d}{|B(0,1)|^{a} r^{(p+a) d}} \int_{0}^{r} \tau^{p d+a d-1}\left(\frac{1}{|B(0,1)|^{p} \tau^{p d}} \int_{B(0, \tau)} g_{R}(t) d t\right) d \tau \\
= & \frac{a d}{|B(0,1)|^{a} r^{(p+a) d}} \int_{0}^{r} \tau^{p d+a d-1}\left(\frac{1}{|B(0, \tau)|^{p}} \int_{B(0, \tau)} g_{R}(t) d t\right) d \tau \\
= & \frac{a d}{|B(0,1)|^{a} r^{(p+a) d}} \int_{0}^{r} \tau^{p d+a d-1}\left(\frac{1}{|B(0, \tau)|^{p}} \int_{B(0, \tau)} g_{R}(t) d t-C_{p}\left(g_{R}\right)\right) d \tau \\
& \quad+\frac{a d}{|B(0,1)|^{a} r^{(p+a) d}}\left(\int_{0}^{r} \tau^{p d+a d-1} d \tau\right) C_{p}\left(g_{R}\right)
\end{aligned}
$$


The second term of (3.6) is equal to

$$
\frac{a d}{|B(0,1)|^{a} r^{(p+a) d}} \frac{1}{(p+a) d} r^{p d+a d} C_{p}\left(g_{R}\right)=\frac{1}{|B(0,1)|^{a}} \frac{a}{p+a} C_{p}\left(g_{R}\right)
$$

and the first term of (3.6) is majorized, in modulus, by

$$
\frac{a d}{|B(0,1)|^{a} r^{(p+a) d}} \int_{0}^{r} \tau^{(p+a) d-1}\left|\frac{1}{|B(0, \tau)|^{p}} \int_{B(0, \tau)} g_{R}(t) d t-C_{p}\left(g_{R}\right)\right| d \tau
$$

Let $\varepsilon>0$ be given. Then since by assumption

$$
\lim _{\tau \rightarrow \infty}\left|\frac{1}{|B(0, \tau)|^{p}} \int_{B(0, \tau)} g_{R}(t) d t-C_{p}\left(g_{R}\right)\right|=0
$$

there exists $t_{\varepsilon}$ such that, for any $\tau \geq t_{\varepsilon}$

$$
\left|\frac{1}{|B(0, \tau)|^{p}} \int_{B(0, \tau)} g_{R}(t) d t-C_{p}\left(g_{R}\right)\right| \leq \varepsilon
$$

Moreover, since $g_{R}$ is locally integrable, the map

$$
\tau \in \mathbb{R}_{+} \longmapsto \frac{1}{|B(0, \tau)|^{p}} \int_{B(0, \tau)} g_{R}(t) d t
$$

is continuous, therefore there exists a constant $C>0$ such that for all $\tau \leq t_{\varepsilon}$

$$
\left|\frac{1}{|B(0, \tau)|^{p}} \int_{B(0, \tau)} g_{R}(t) d t-C_{p}\left(g_{R}\right)\right| \leq C
$$

Therefore, by splitting the integral in $\tau$ in (3.8) into the two pieces, (3.8) is majorized by

$$
\begin{aligned}
& \frac{a d}{|B(0,1)|^{a} r^{(p+a) d}}\left(C \int_{0}^{t_{\varepsilon}} \tau^{(p+a) d-1} d \tau+\varepsilon \int_{t_{\varepsilon}}^{r} \tau^{(p+a) d-1} d \tau\right) \\
& \leq \frac{a d}{|B(0,1)|^{a}}\left(\frac{1}{(p+a) d}\left(\frac{t_{\varepsilon}}{r}\right)^{(p+a) d} C+\varepsilon \frac{1}{r^{(p+a) d}} \int_{0}^{r} \tau^{(p+a) d-1} d \tau\right) \\
& \leq \frac{a}{|B(0,1)|^{a}(p+a)}\left(\left(\frac{t_{\varepsilon}}{r}\right)^{(p+a) d} C+\varepsilon\right)
\end{aligned}
$$

and so

$$
\begin{aligned}
& \lim _{r \rightarrow \infty} \frac{a d}{|B(0,1)|^{a} r^{(p+a) d}} \int_{0}^{r} \tau^{(p+a) d-1}\left|\frac{1}{|B(0, \tau)|^{p}} \int_{B(0, \tau)} g_{R}(t) d t-C_{p}\left(g_{R}\right)\right| d \tau \\
& \quad \leq \lim _{r \rightarrow \infty} \frac{a}{|B(0,1)|^{a}(p+a)}\left(\left(\frac{t_{\varepsilon}}{r}\right)^{(p+a) d} C+\varepsilon\right) \\
& \quad \leq \frac{a}{|B(0,1)|^{a}(p+a)} \varepsilon
\end{aligned}
$$


from which, since $\varepsilon>0$ is arbitrary,

$$
\lim _{r \rightarrow \infty} \frac{a d}{|B(0,1)|^{a} r^{(p+a) d}} \int_{0}^{r} \tau^{p d+a d-1}\left(\frac{1}{|B(0, \tau)|^{p}} \int_{B(0, \tau)} g_{R}(t) d t-C_{p}\left(g_{R}\right)\right) d \tau=0
$$

Hence by (3.5), (3.6), (3.7) and (3.9), we have

$$
\lim _{r \rightarrow \infty} \frac{1}{|B(0, r)|^{p+a}} \int_{B(0, r)}\left(r^{a d}-|t|^{a d}\right) g_{R}(t) d t=\frac{1}{|B(0,1)|^{a}} \frac{a}{p+a} C_{p}\left(g_{R}\right) .
$$

Therefore, by (3.3) and (3.4), we have

$$
\begin{aligned}
\lim _{r \rightarrow \infty} \frac{1}{|B(0, r)|^{p+a}} \int_{B(0, r)}|t|^{a d} g_{R}(t) d t & =\frac{1}{|B(0,1)|^{a}} C_{p}\left(g_{R}\right)-\frac{1}{|B(0,1)|^{a}} \frac{a}{p+a} C_{p}\left(g_{R}\right) \\
& =\frac{1}{|B(0,1)|^{a}} \frac{p}{p+a} C_{p}\left(g_{R}\right),
\end{aligned}
$$

which implies (3.2) as desired.

Remark 3.2. Notice that, for $p=0$, the main assumption of Theorem 3.1, i.e. (3.1), is not reduced to the assumption of the usual Cesàro theorem (Theorem 2.3), i.e. (2.8).

\section{A Converse Version of Higher Order Cesàro Theorem}

The following theorem gives the converse of Theorem 3.1.

Theorem 4.1. If, for some $p \in \mathbb{R}_{+}^{*}$, some $a \in \mathbb{R}_{+}$and some $f \in L_{\text {loc }}^{1}\left(\mathbb{R}^{d}\right)$, the limit

$$
C_{p+a}(f):=\lim _{r \rightarrow \infty} \frac{1}{|B(0, r)|^{p+a}} \int_{B(0, r)} f(t) d t
$$

exists in $\mathbb{C}$ and $|\cdot|^{-a d} f(\cdot) \in L_{\mathrm{loc}}^{1}\left(\mathbb{R}^{d}\right)$, then it holds that

$$
\lim _{r \rightarrow \infty} \frac{1}{|B(0, r)|^{p}} \int_{B(0, r)}|t|^{-a d} f(t) d t=\frac{p+a}{p}|B(0,1)|^{a} C_{p+a}(f)
$$

in the sense that the limit exists and the equality holds.

Proof. The proof is similar to the one of Theorem 3.2. Let $p \in \mathbb{R}_{+}^{*}, a \in \mathbb{R}_{+}$and $f \in L_{\text {loc }}^{1}\left(\mathbb{R}^{d}\right)$ be such that (4.1) holds. Then we have

$$
\begin{aligned}
\frac{1}{|B(0, r)|^{p}} \int_{B(0, r)}|t|^{-a d} f(t) d t= & \frac{1}{|B(0, r)|^{p}} r^{-a d} \int_{B(0, r)} f(t) d t \\
& -\frac{1}{|B(0, r)|^{p}} \int_{B(0, r)}\left(r^{-a d}-|t|^{-a d}\right) f(t) d t
\end{aligned}
$$


On the one hand, with the same notations of Theorem 3.2

$$
\begin{gathered}
-\frac{1}{|B(0, r)|^{p}} \int_{B(0, r)}\left(r^{-a d}-|t|^{-a d}\right) f(t) d t \\
=-\frac{1}{|B(0,1)|^{p} r^{p d}} \int_{0}^{r}\left(r^{-a d}-s^{-a d}\right) s^{d-1}\left(\int_{\Theta} \hat{f}(s, \hat{\theta}) d \hat{\theta}\right) d s \\
=\frac{a d}{|B(0,1)|^{p} r^{p d}} \int_{0}^{r} s^{d-1}\left(\int_{s}^{r} \tau^{-a d-1} d \tau\right)\left(\int_{\Theta} \hat{f}(s, \hat{\theta}) d \hat{\theta}\right) d s \\
=\frac{a d}{|B(0,1)|^{p} r^{p d}} \int_{0}^{r}\left[\int_{0}^{\tau} s^{d-1}\left(\int_{\Theta} \hat{f}(s, \hat{\theta}) d \hat{\theta}\right) d s\right] \tau^{-a d-1} d \tau= \\
=\frac{a d|B(0,1)|^{a}}{r^{p d}} \int_{0}^{r} \tau^{p d-1}\left(\frac{1}{|B(0, \tau)|^{p+a}} \int_{B(0, \tau)} f(t) d t\right) d \tau \\
=\frac{a d|B(0,1)|^{a}}{r^{p d}} \int_{0}^{r} \tau^{p d-1}\left(\frac{1}{|B(0, \tau)|^{p+a}} \int_{B(0, \tau)} f(t) d t-C_{p+a}(f)\right) d \tau \\
+\frac{a d|B(0,1)|^{a}}{r^{p d}}\left(\int_{0}^{r} \tau^{p d-1} d \tau\right) C_{p+a}(f) .
\end{gathered}
$$

Therefore, by (4.3), (4.4) and (4.5) the following identity holds:

$$
\frac{1}{|B(0, r)|^{p}} \int_{B(0, r)}|t|^{-a d} f(t) d t=k_{1}(r)+k_{2}(r)+k_{3}(r)
$$

where

$$
\begin{aligned}
& k_{1}(r)=\frac{1}{|B(0, r)|^{p}} r^{-a d} \int_{B(0, r)} f(t) d t \\
& k_{2}(r)=\frac{a d|B(0,1)|^{a}}{r^{p d}}\left(\int_{0}^{r} \tau^{p d-1} d \tau\right) C_{p+a}(f) \\
& k_{3}(r)=\frac{a d|B(0,1)|^{a}}{r^{p d}} \int_{0}^{r} \tau^{p d-1}\left(\frac{1}{|B(0, \tau)|^{p+a}} \int_{B(0, \tau)} f(t) d t-C_{p+a}(f)\right) d \tau
\end{aligned}
$$

Then by (4.6) and the assumption we obtain that

$$
\lim _{r \rightarrow \infty} k_{1}(r)=|B(0,1)|^{a} \lim _{r \rightarrow \infty} \frac{1}{|B(0, r)|^{p+a}} \int_{B(0, r)} f(t) d t=|B(0,1)|^{a} C_{p+a}(f)
$$

and from (4.7), we have

$$
k_{2}(r)=\frac{a d|B(0,1)|^{a}}{r^{p d}}\left(\int_{0}^{r} \tau^{p d-1} d \tau\right) C_{p+a}(f)=\frac{a}{p}|B(0,1)|^{a} C_{p+a}(f)
$$

Also, by the same arguments used for the proof of (3.9), we prove that $\lim _{t \rightarrow \infty} k_{3}(t)=0$. 
Therefore, from the above arguments it follows that

$$
\begin{aligned}
\lim _{r \rightarrow \infty} \frac{1}{|B(0, r)|^{p}} \int_{B(0, r)}|t|^{-a d} f(t) d t & =\lim _{r \rightarrow \infty}\left(k_{1}(r)+k_{2}(r)+k_{3}(r)\right) \\
& =|B(0,1)|^{a} C_{p+a}(f)+\frac{a}{p}|B(0,1)|^{a} C_{p+a}(f) \\
& =\frac{p+a}{p}|B(0, r)|^{a} C_{p+a}(f)
\end{aligned}
$$

which is the desired result.

Remark 4.2. The statement of Theorem 4.1 does not hold for $p=0$. For example if

$$
f(t)= \begin{cases}1 & (|t| \geq M) \\ 0 & (|t|<M)\end{cases}
$$

for some $M>0$, then

$$
\lim _{r \rightarrow \infty} \frac{1}{r} \int_{-r}^{r} f(t) d s=\lim _{r \rightarrow \infty} \frac{2(r-M)}{r}=2 \quad\left(=C_{1}(f)\right)
$$

but, we have

$$
\lim _{r \rightarrow \infty} \int_{-r}^{r} \frac{1}{|t|} f(t) d t=\lim _{r \rightarrow \infty} 2(\log r-\log M)=+\infty
$$

\section{Reduction to Discrete Cesàro Theorems}

In this section, by reducing from continuous to discrete case, we show how, from the previous theorems, one can obtain the corresponding statements for sequences.

Sequences of complex numbers, i.e. elements of $\mathbb{C}^{\infty}$, are identified with functions in $L^{1}(\mathbb{R})$ which are constant in the intervals $[k, k+1)(k \in \mathbb{Z})$. More precisely, we define the embedding $c: \mathbb{C}^{\infty} \ni a \mapsto c_{a} \in L_{l o c}^{1}(\mathbb{R})$ by

$$
c_{a}(t):= \begin{cases}a_{n}, & n \in \mathbb{N}, t \in[n, n+1), \\ 0, & t<1 .\end{cases}
$$

The action of the functionals $\widetilde{C}_{p}$ on $\mathbb{C}^{\infty}$ is defined by

$$
\begin{gathered}
\widetilde{C}_{p}(a):=2^{p} C_{p}\left(c_{a}\right):=\lim _{n \rightarrow+\infty} \frac{1}{n^{p}} \sum_{k=1}^{n} a_{k} \\
\operatorname{Dom}\left(\widetilde{C}_{p}\right):=\left\{a \in \mathbb{C}^{\infty}: \lim _{n \rightarrow \infty} \frac{1}{n^{p}} \sum_{k=1}^{n} a(k) \text { exists in } \mathbb{C}\right\}
\end{gathered}
$$

Then clearly for any $p \geq 0$,

$$
a \in \operatorname{Dom}\left(\widetilde{C}_{p}\right) \Leftrightarrow c_{a} \in \operatorname{Dom}\left(C_{p}\right)
$$


Let $Q$ be the locally integrable function on $\mathbb{R}$ defined by

$$
Q(t)= \begin{cases}\frac{[|t|]}{|t|}, & t \geq 1 \\ 0, & t<1\end{cases}
$$

where $[|t|]$ is the largest integer smaller than $|t|$. For our convenience, we understand that $\infty \cdot 0=0$.

Lemma 5.1. Let $a \in \operatorname{Dom}\left(\widetilde{C}_{p}\right)$. Then for any $\beta \in \mathbb{R}$, it holds that

$$
\lim _{r \rightarrow \infty} \frac{1}{r^{p}} \int_{1}^{r}\left[\left(\frac{[|t|]}{|t|}\right)^{\beta}-1\right] c_{a}(t) d t=0
$$

Proof. Let $\beta \in \mathbb{R}$ be given. Then we obtain that

$$
\begin{array}{r}
\lim _{r \rightarrow \infty} \frac{1}{r^{p}} \int_{1}^{r}\left[\left(\frac{[|t|]}{|t|}\right)^{\beta}-1\right] c_{a}(t) d t=\lim _{r \rightarrow \infty} \frac{1}{r^{p}} \sum_{k=1}^{[r]-1} a_{k}\left(\int_{k}^{k+1}\left[\left(\frac{k}{t}\right)^{\beta}-1\right] d t\right) \\
+\lim _{r \rightarrow \infty} \frac{a_{[r]}}{r^{p}} \int_{[r]}^{r}\left[\left(\frac{[r]}{t}\right)^{\beta}-1\right] d t
\end{array}
$$

Then we can easily see that the second term of the right hand side of (5.1) is zero and the first term of the right hand side of (5.1) coincides with the following:

$$
\lim _{N \rightarrow \infty} \frac{1}{N^{p}} \sum_{k=1}^{N} a_{k} b_{k}, \quad b_{k}=\int_{k}^{k+1}\left[\left(\frac{k}{t}\right)^{\beta}-1\right] d t
$$

Therefore, we have

$$
\lim _{r \rightarrow \infty} \frac{1}{r^{p}} \int_{1}^{r}\left[\left(\frac{[|t|]}{|t|}\right)^{\beta}-1\right] c_{a}(t) d t=\lim _{N \rightarrow \infty} \frac{1}{N^{p}} \sum_{k=1}^{N} a_{k} b_{k}
$$

Then the Abel identity implies that

$$
\begin{aligned}
\lim _{N \rightarrow \infty} \frac{1}{N^{p}} \sum_{k=1}^{N} a_{k} b_{k} & =\lim _{N \rightarrow \infty} \frac{1}{N^{p}}\left(\sum_{k \leq N} a_{k}\right) b_{N}-\lim _{N \rightarrow \infty} \frac{1}{N^{p}} \sum_{k \leq N-1}\left(b_{k+1}-b_{k}\right)\left(\sum_{r \leq k} a_{r}\right) \\
& =-\lim _{N \rightarrow \infty} \frac{1}{N^{p}} \sum_{k \leq N-1}\left(b_{k+1}-b_{k}\right) k^{p}\left(\frac{1}{k^{p}} \sum_{r \leq k} a_{r}\right)
\end{aligned}
$$

On the other hand, by direct computation we can prove that $\lim _{k \rightarrow \infty} k\left(b_{k+1}-b_{k}\right)=0$. Let $\left\{d_{n}\right\}_{n=1}^{\infty}$ be a sequence given by

$$
d_{n}=-n\left(b_{n+1}-b_{n}\right)\left(\frac{1}{n^{p}} \sum_{r \leq n} a_{r}\right), \quad n \in \mathbb{N}
$$


Then we have $\lim _{n \rightarrow \infty} d_{n}=0 \widetilde{C}_{p}(a)=0$, i.e., for any $\epsilon>0$ there exists a number $N_{0}$ such that $\left|d_{n}\right|<\epsilon$ if $n \geq N_{0}$. Using this sequence (5.2) is represented as follows:

$$
\begin{aligned}
\lim _{r \rightarrow \infty} \frac{1}{r^{p}} \int_{1}^{r}\left[\left(\frac{[|t|]}{|t|}\right)^{\beta}-1\right] c_{a}(t) d t & =\lim _{N \rightarrow \infty} \frac{1}{N^{p}} \sum_{k=1}^{N} a_{k} b_{k} \\
& =-\lim _{N \rightarrow \infty} \frac{1}{N^{p}} \sum_{k \leq N-1}\left(b_{k+1}-b_{k}\right) k^{p}\left(\frac{1}{k^{p}} \sum_{r \leq k} a_{r}\right) \\
& =\lim _{N \rightarrow \infty} \frac{1}{N^{p}} \sum_{k \leq N-1} k^{p-1} d_{k}
\end{aligned}
$$

Therefore we obtain that

$$
\begin{array}{r}
\left|\lim _{r \rightarrow \infty} \frac{1}{r^{p}} \int_{1}^{r}\left[\left(\frac{[|t|]}{|t|}\right)^{\beta}-1\right] c_{a}(t) d t\right| \leq \lim _{N \rightarrow \infty} \frac{1}{N^{p}} \sum_{k \leq N-1} k^{p-1}\left|d_{k}\right| \\
=\lim _{N \rightarrow \infty} \frac{1}{N^{p}} \sum_{k=0}^{N_{0}-1} k^{p-1}\left|d_{k}\right|+\lim _{N \rightarrow \infty} \frac{1}{N^{p}} \sum_{k=N_{0}}^{N-1} k^{p-1}\left|d_{k}\right| \leq \lim _{N \rightarrow \infty} \frac{1}{N^{p}} \sum_{k=N_{0}}^{N-1} k^{p-1}\left|d_{k}\right| \leq C \epsilon
\end{array}
$$

with a constant $C$ independent on $N$. Since $\epsilon$ is arbitrary, this gives the proof of the assertion.

Lemma 5.2. Let $a \in \operatorname{Dom}\left(\widetilde{C}_{p}\right)$. Then for any $\beta \in \mathbb{R}, Q^{\beta} c_{a} \in \operatorname{Dom}\left(C_{p}\right)$ and

$$
C_{p}\left(Q^{\beta} c_{a}\right)=C_{p}\left(c_{a}\right)=2^{-p} \widetilde{C}_{p}(a) .
$$

Proof. For given $\beta \in \mathbb{R}$, by applying Lemma 5.1, we obtain that

$$
\begin{aligned}
C_{p}\left(Q^{\beta} c_{a}\right) & =\lim _{r \rightarrow \infty} \frac{1}{|B(0, r)|^{p}} \int_{B(0, r)}\left(\frac{[|t|]}{|t|}\right)^{\beta} c_{a}(t) d t \\
& =\lim _{r \rightarrow \infty} \frac{1}{|B(0, r)|^{p}} \int_{B(0, r)}\left[\left(\frac{[|t|]}{|t|}\right)^{\beta}-1\right] c_{a}(t) d t+C_{p}\left(c_{a}\right) \\
& =C_{p}\left(c_{a}\right)
\end{aligned}
$$

which gives the proof.

Denote $q$ the identity function on $\mathbb{R}, q(t)=t$ for any $t \in \mathbb{R}$.

Lemma 5.3. Let $a \in \operatorname{Dom}\left(\widetilde{C}_{p}\right)$ and $\beta \in \mathbb{R}$. Then $|q|^{\beta} Q^{\beta} c_{a} \in \operatorname{Dom}\left(C_{p}\right)$ and

$$
C_{p}\left(|q|^{\beta} Q^{\beta} c_{a}\right)=2^{-p} \widetilde{C}_{p}\left(q_{d}^{\beta} a\right)
$$

where $q_{d}$ is the discretization of the multiplication operator $q$, i.e., $\left(q_{d}^{\beta} a\right)(n)=n^{\beta} a_{n}$ for $a \in \mathbb{C}^{\infty}$. 
Proof. We obtain that

$$
\begin{aligned}
C_{p}\left(|q|^{\beta} Q^{\beta} c_{a}\right) & =\lim _{r \rightarrow \infty} \frac{1}{(2 r)^{p}} \int_{-r}^{r}|q|(t)^{\beta} Q(t)^{\beta} c_{a}(t) d t \\
& =\lim _{r \rightarrow \infty} \frac{1}{(2 r)^{p}} \int_{-r}^{r}|t|^{\beta}\left(\frac{[|t|]}{|t|}\right)^{\beta} c_{a}(t) d t \\
& =\lim _{n \rightarrow \infty} \frac{1}{(2 n)^{p}} \int_{1}^{n}[|t|]^{\beta} c_{a}(t) d t \\
& =2^{-p} \lim _{n \rightarrow \infty} \frac{1}{n^{p}} \sum_{k=1}^{n} k^{\beta} a_{k} \\
& =2^{-p} \widetilde{C}_{p}\left(q_{d}^{\beta} a\right)
\end{aligned}
$$

which implies the proof.

By Lemma 5.2, the continuous higher order Cesàro theorem reduces the higher order Cesàro theorem for sequences. Then the following theorems follow from Theorems 3.1 and 4.1 .

Theorem 5.4. ([10], [5]) Let the sequence $a=\left(a_{n}\right)_{n=1}^{\infty} \in \mathbb{C}^{\infty}$ be such that, for some $p>0$ the limit

$$
\lim _{N \rightarrow \infty} \frac{1}{N^{p}} \sum_{n=1}^{N} a_{n}=: C_{p}(a)
$$

exists. Then for each $\alpha \in \mathbb{R}_{+}$, one has

$$
\lim _{N \rightarrow \infty} \frac{1}{N^{p+\alpha}} \sum_{n=1}^{N} n^{\alpha} a_{n}=\frac{p}{p+\alpha} C_{p}(a)
$$

in the sense that the limit on the left hand side exists and the equality holds.

Theorem 5.5. ([10], [5]) Let $p>0, \alpha \geq 0$ and let $a=\left(a_{n}\right)_{n=1}^{\infty}$ be a sequence in $\mathbb{C}^{\infty}$ such that the limit

$$
\lim _{N \rightarrow \infty} \frac{1}{N^{p+\alpha}} \sum_{n=1}^{N} a_{n}=: C_{p+\alpha}(a)
$$

exists. Then

$$
\lim _{N \rightarrow \infty} \frac{1}{N^{p}} \sum_{n=1}^{N} n^{-\alpha} a_{n}=\frac{p+\alpha}{p} C_{p+\alpha}(a)
$$

\section{References}

[1] L. Accardi, A. Barhoumi and H. Ouerdiane: A Quantum approach to Laplace operators, Infin. Dimens. Anal. Quantum Probab. Relat. Top. 9 (2006), 215-248.

[2] L. Accardi, P. Gibilisco and I. V. Volovich: Yang-Mills gauge fields as harmonic functions for the Lévy Laplacian, Russian J. Math. Phys. 2 (1994), 235-250. 
[3] L. Accardi, U. C. Ji and K. Saitô: Exotic Laplacians and associated stochastic processes, Infin. Dimens. Anal. Quantum Probab. Relat. Top. 12 (2009), 1-19.

[4] L. Accardi, U. C. Ji, K. Saitô: Exotic Laplacians and derivatives of white noise, Infin. Dimens. Anal. Quantum Probab. Relat. Top. 14 (2011), 1-14.

[5] L. Accardi, U. C. Ji and K. Saitô: The exotic (higher order Lévy) Laplacians generate the Markov processes given by distribution derivatives of white noise, Infin. Dimens. Anal. Quantum Probab. Relat. Top. 16 (2013), no. 3, 1350020.

[6] L. Accardi, H. Ouerdiane and O. G. Smolyanov: Lévy Laplacian acting on operators, Russian J. Math. Phys. 10 (2003), 359-380.

[7] L. Accardi, P. Roselli and O. G. Smolyanov: Brownian motion generated by the Lévy Laplacian, Math. Notes 54 (1993), 1174-1177.

[8] L. Accardi and O. G. Smolyanov: On Laplacians and traces, Confer. Sem. Mat. Univ. Bari No. 250 (1993).

[9] L. Accardi and O. G. Smolyanov: Classical and nonclassical Lévy Laplacians, Dokl. Math. 76 (2007), no. 3, 801-805.

[10] L. Accardi and O. G. Smolyanov: Generalized Levy Laplacians and Cesàro means, Doklady Akademii Nauk 424 (2009), 583-587.

[11] D. M. Chung, U. C. Ji and K. Saitô: Cauchy problems associated with the Lévy Laplacian in white noise analysis, Infin. Dimens. Anal. Quantum Probab. Relat. Top. 2 (1999), 131-153.

[12] M. N. Feller: Infinite-dimensional elliptic equations and operators of Lévy type, Russian Math. Surveys 41 (1986), 119-170.

[13] G. M. Fikhtengol'ts: "A Course in Differential and Integral Calculus," Vol. 2 [in Russian], Moscow, 1959.

[14] U. C. Ji, N. Obata and H. Ouerdiane: Quantum Lévy Laplacian and associated heat equation, J. Funct. Anal. 249 (2007), 31-54.

[15] U. C. Ji and K. Saitô: A similarity between the Gross Laplacian and the Lévy Laplacian, Infin. Dimens. Anal. Quantum Probab. Relat. Top. 10 (2007), 261-276.

[16] H.-H. Kuo, N. Obata and K. Saitô: Lévy Laplacian of generalized functions on a nuclear space, J. Funct. Anal. 94 (1990), 74-92.

[17] P. Lévy: "Leçons d'Analyse Fonctionnelle," Gauthier-Villars, Paris, 1922.

[18] P. Lévy: "Problemes Concrets d'Analyse Fonctionnelles," Gauthier-Villars, Paris, 1951. 
[19] N. Obata: Quadratic quantum white noises and Lévy Laplacian, Nonlinear Anal. 47 (2001), 2437-2448.

[20] K. Saitô: A stochastic process generated by the Lévy Laplacian, Acta Appl. Math. 63 (2000), 363-373. 